\title{
Post-Traumatic Stress Disorders on Pediatric Orthopedic Injuries
}

\section{Ming-Sheng Tan* and Liang Dong}

The Department of Orthopedics Surgery, China-Japan Friendship Hospital, Beijing 100029, People's Republic of China

Post-traumatic Stress Disorders (PTSD) is a mental disorder that happens on people who experience a catastrophic traumatic event, they show helplessness, fear and other emotions, and the symptom of repeated traumatic emotional numbing and avoidance of traumatic events and increased awareness [1]. Xiang et al. [2] reported that $89 \%$ of the injuries were orthopedic traumas with lower limb fracture through studying 119 children after the 2008 Wen-Chuan earthquake in China. A serious trauma often includes the orthopedic injuries, which has a serious impact on the work and daily life after the actual extremities trauma or dysfunction of extremities induced by spinal injury. Because of the immaturity of cognitive capacity and lack of social experience, the rate of pediatric PTSD is high. As orthopaedic surgeons, we should be aware of the psychologic effects, as well as child's physical injuries.

Pynoos et al. [3] reported that the rate of pediatric PTSD was $69.0 \%$ 18 months after an earthquake. The rate of PTSD among the children after a massive earthquake struck China's Wen-chuan was the $78.3 \%$ in the study of Liu et al [4]. Giannopoulou et al. [5] showed that the symptom of PTSD was associated with age and gender after studying 2037 children undergoing the Athens earthquake; more PTSD, anxiety and depressive symptoms occurred in Girls, and younger children reported significantly more PTSD and anxiety symptoms than the older ones. In a study of 400 children with minor orthopaedic injuries, 33\% of patients met the symptoms of a high level of PTSD at 1 month after the trauma [6]. Hence, it is important and meaningful to analyze the diagnosis, prevention and treatment of PTSD induced by orthopedic injuries [7].

If there were no prevention of PTSD or timely diagnosis and treatmet for children after a serious orthopedic trauma, the future treatment process will become more difficult [8]. It is important that children have a correct attitude for trauma and actively participate in the prevention and treatment of PTSD. The main treatment of pediatric PTSD includes drug therapy and psychological-social support. Strawn [9] proposed escalation drug therapy for pediatric PTSD. Compared with drug therapy, early effective social support and psychological intervention plays a more important role, which can avoid the occurrence of PTSD through relieving stress, encouraging the self-rehabilitation and social adjustment function [10-12]. The study of Goenjian et al. on pediatric PTSD 5 years after a disaster [13] reported that the rate of PTSD and depression after the positive psychological intervention was lower than the non-treatment group. Dana [14] showed that pediatric PTSD also appeared in a variety of sugeries. Hence, clinicians should pay more attention on postoperative prevention of PTSD. Additionally, whether low- or high-energy orthopaedic injury, the prevalence of PTSD was not significant in the study of Wallace [15]; hence, we also pay attention to the children who sustained a low-energy fracture treated non-operatively.

In recent years, there are many studies on PTSD, but, however, only limited information exists on diagnosis and treatment of pediatric PTSD. In addition, whereas a main focus lies on the treatment and rehabilitation itself, little attention is paid to the pathogenesis and psychological, social intervention of PTSD. Consequently, the Journal of Trauma and Treatment provides a meaningful platform for analyzing the issue of PTSD, we can further discuss and communicate with many experts about the issue of pediatric PTSD.

\section{References}

1. American Psychiatric Association American Psychiatric Association (1994) Task Force on DSM-IV. Diagnostic and statistical manual of mental disorders: DSM-IV[M]. Amer Psychiatric Pub Inc.

2. Xiang B, Cheng W, Liu J, Huang L, Li Y, et al. (2009) Triage of pediatric injuries after the 2008 Wen-Chuan earthquake in China. J Pediatr Surg 44: 2273-2277.

3. Pynoos RS, Goenjian A, Tashjian M, Karakashian M, Manjikian R, et al. (1993) Post-traumatic stress reactions in children after the 1988 Armenian earthquake. Br J Psychiatry 163: 239-247.

4. Liu XX, Xiong GY, Ma DC, Dong YQ, Ye YL, et al. (2009) [Relationship between post-traumatic stress disorder and perceived social support among middle school students in Wenchuan earthquake-stricken]. Zhonghua Liu Xing Bing Xue Za Zhi 30: 1017-1020.

5. Giannopoulou I, Strouthos M, Smith P, Dikaiakou A, Galanopoulou V, et al (2006) Post-traumatic stress reactions of children and adolescents exposed to the Athens 1999 earthquake. Eur Psychiatry 21: 160-166.

6. Sanders MB1, Starr AJ, Frawley WH, McNulty MJ, Niacaris TR (2005) Posttraumatic stress symptoms in children recovering from minor orthopaedic injury and treatment. J Orthop Trauma 19: 623-628.

7. Andruszkow H (2012) Impacts on Long-Term Outcome after Major TraumaTraumatic Brain and Orthopedic Injuries[J]. J Trauma Treat S 2: 2167-1222.

8. Shalev AY, Bonne O, Eth S (1996) Treatment of posttraumatic stress disorder: a review. Psychosom Med 58: 165-182.

9. Strawn JR, Keeshin BR, DelBello MP, Geracioti TD Jr, Putnam FW (2010) Psychopharmacologic treatment of posttraumatic stress disorder in children and adolescents: a review. J Clin Psychiatry 71: 932-941.

10. Smith P, Yule W, Perrin S, Tranah T, Dalgleish T, et al. (2007) Cognitivebehavioral therapy for PTSD in children and adolescents: a preliminary randomized controlled trial. J Am Acad Child Adolesc Psychiatry 46: 10511061.

11. Bisson J, Andrew M (2007) Psychological Treatment of Post-traumatic Stress Disorder (PTSD) Wiley.

12. Lavie $P$ (2001) Sleep disturbances in the wake of traumatic events. N Engl J Med 345: 1825-1832.

13. Goenjian AK, Walling D, Steinberg AM, Karayan I, Najarian LM, et al. (2005) A prospective study of posttraumatic stress and depressive reactions among treated and untreated adolescents 5 years after a catastrophic disaster. Am J Psychiatry 162: 2302-2308.

14. Connolly D, McClowry S, Hayman L, Mahony L, Artman M (2004) Posttraumatic stress disorder in children after cardiac surgery. J Pediatr 144: 480-484.

15. Wallace M, Puryear A, Cannada LK (2013) An evaluation of posttraumatic stress disorder and parent stress in children with orthopaedic injuries. J Orthop Trauma 27: e38-41.

*Corresponding author: Mingsheng Tan, The Department of Orthopedics Surgery, China-Japan Friendship Hospital, Beijing 100029, People's Republic of China, Tel: +86 10 84205011; Fax: +86 10 84205011; E-mail: zrtanms@sina.com

Received January 07, 2014; Accepted February 26, 2014; Published February 28, 2014

Citation: Tan MS, Dong L (2014) Post-Traumatic Stress Disorders on Pediatric Orthopedic Injuries. J Trauma Treat S4: 012. doi:10.4172/2167-1222.S4-012

Copyright: (c) 2014 Tan MS, et al. This is an open-access article distributed under the terms of the Creative Commons Attribution License, which permits unrestricted use, distribution, and reproduction in any medium, provided the original author and source are credited. 